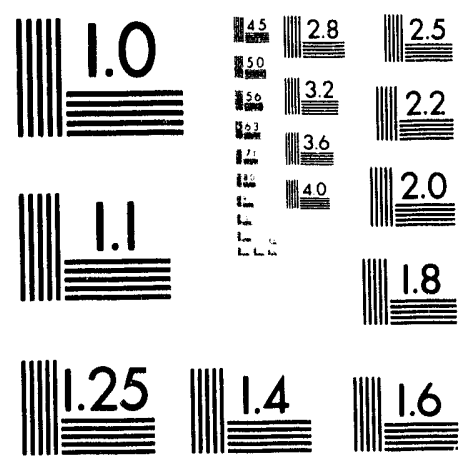



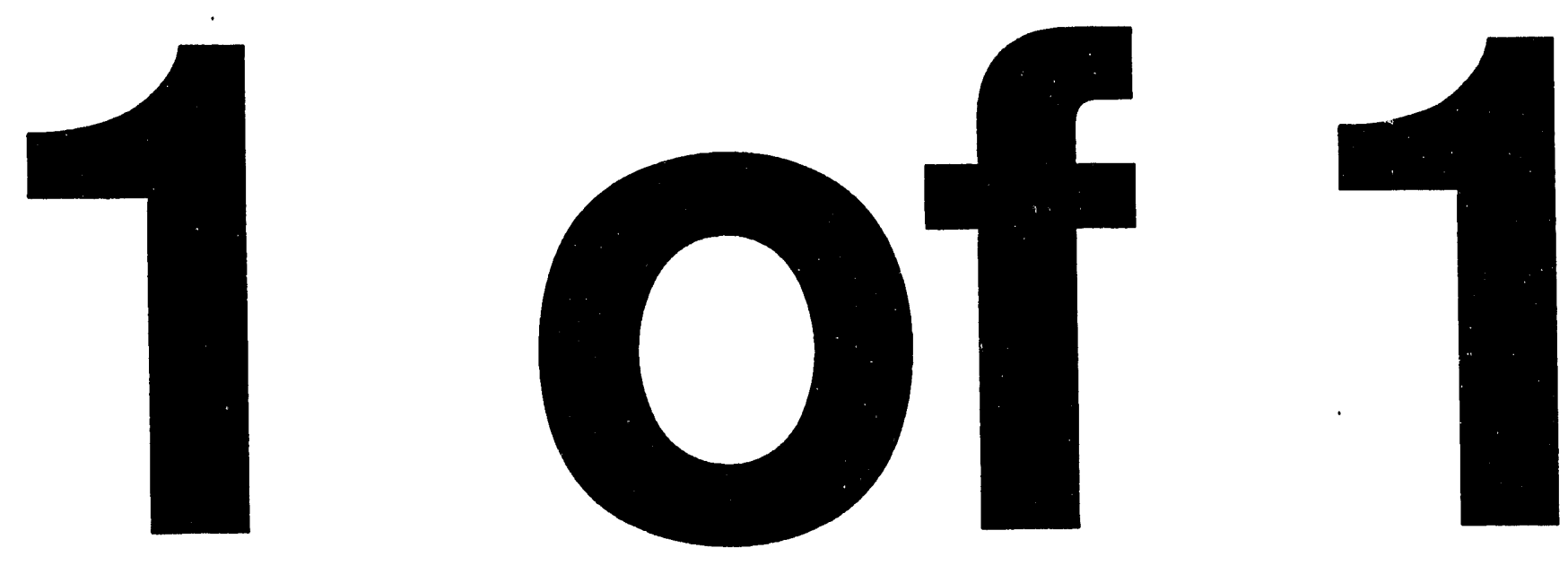


\title{
Low Energy X-ray Response of Ge Detectors with Amorphous Ge Entrance Contacts
}

\author{
P.N. Luke, C.S. Rossington and M.F. Wesela \\ Engineering Division \\ Lawrence Berkeley Laboratory \\ University of California, Berkeley CA 94720
}

October 1993

This work was supported by the Director, Office of Energy Research, Otfice of Biological and Environmental Research, Analytical Technology Division, of the U.S. Department of Energy under Contract Number DE-AC03-76SF00098. 


\title{
Low Energy X-ray Response of Ge Detectors with Amorphous Ge Entrance Contacts
}

\author{
P.N. Luke, C.S. Rossington and M.F. Wesela \\ Engineering Division, Lawrence Berkeley Laboratory ${ }^{1}$ \\ University of California, Berkeley, CA 94720
}

\begin{abstract}
The low energy $x$-ray response of Ge detectors with amorphous $\mathrm{Ge}$ entrance contacts has been evaluated. The spectral background due to near contact incomplete charge collection was found to consist of two components: a low level component which is insensitive to applied voltage and a high level step-like component which is voltage dependent. At high operating voltages, the high level component can be completely suppressed, resulting in background levels which are much lower than those previously observed using $\mathrm{Ge}$ detectors with Pd surface barrier or B ion implanted contacts, and which also compare favorably to those obtained with $\mathrm{Si}(\mathrm{Li}) \mathrm{x}$-ray detectors. The response of these detectors to ${ }^{55} \mathrm{Fe}$ and $1.77 \mathrm{keV} x$-rays is shown. A qualitative explanation of the origins of the observed background components is presented.
\end{abstract}

\section{INTRODUCTION}

Ge detectors with Pd surface barrier or B ion implanted contacts typically exhibit excessive spectral backgrounds when used in low energy $x$-ray spectroscopy. Si detectors are commonly used in this application, but $\mathrm{Ge}$ is often preferable as a detector material due to its higher ausorption coefficiency and therefore its potentially wider $x$-ray energy response range. In addition, $\mathrm{Ge}$ detectors offer intrinsically better energy resolution due to the smaller ionization energy $(2.97 \mathrm{eV}$ per electron-hole pair at $77 \mathrm{~K}$ compared with $3.76 \mathrm{eV}$ for $\mathrm{Si}$ ). For these reasons, it is desirable to develop Ge detectors with good low-energy $x$-ray response characteristics. Recently, sputtered amorphous $\mathrm{Ge}(\mathrm{a}-\mathrm{Ge})$ contacts have been developed as an alternative to conventional contacts for $\mathrm{Ge}$ radiation detectors. Preliminary data indicated that superior low energy $x$-ray performance could be achieved using these contacts [1]. A more detailed study of the low energy $x$-ray response has been made and the resulis are compared to those obtained previously using $\mathrm{Ge}$ detectors with $\mathrm{Pd}$ surface barrier [2] and B implanted contacts [3].

\section{DETECTOR FABRICATION}

The a-Ge contact detectors used in this evaluation were made from high-purity p-type Ge crystals with a top-hat struc-

\footnotetext{
1 We thank N. W. Madden for useful discussions. This work was supported by the Director, Office of Energy Research, Office of Biological and Environmental Research, Analytical Technology Division, of the U.S. Department of Energy under Contract No. DE-AC03-76SF00098.
}

ture shown schematically in Fig.1. The $\mathrm{n}^{+}$contacts were formed by $\mathrm{Li}$ diffusion with a layer of Pd evaporated on top to provide low resistance electrical connections. To produce the aGe contact, the crystal was first given a chemical etch followed by quenching with methanol. Sputter deposition of the a-Ge layer was then carried out in a gas mixture of $93 \% \mathrm{Ar}$ and $7 \% \mathrm{H}_{2}$ at a pressure of $7 \mu \mathrm{m}$. A thin layer of $\mathrm{Au}$ was deposited on top of the a-Ge layer by evaporation. The characteristics of the detectors reported in this paper are given in Table 1, and are representative of typical devices from the numerous ones that were tested.

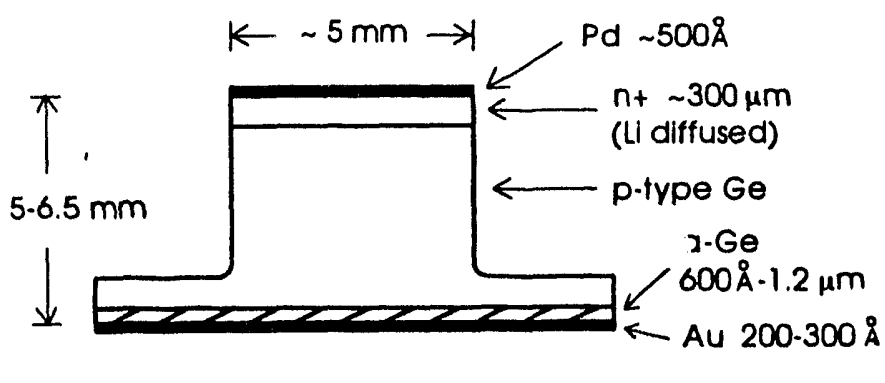

XDL $9310-1448$

Fig. 1. Structure of the a-Ge contact detectors.

Table I

Characteristics of the a-Ge contact detectors

\begin{tabular}{|l|c|c|c|}
\hline Detector No. & $4-3$ & $8-2$ & $6-6$ \\
\hline Detector Thickness & $6.1 \mathrm{~mm}$ & $6.5 \mathrm{~mm}$ & $5.3 \mathrm{~mm}$ \\
\hline Depletion Voltage & $440 \mathrm{~V}$ & $400 \mathrm{~V}$ & $50 \mathrm{~V}$ \\
\hline a-Ge Thickness & $600 \AA$ & $1200 \AA$ & $1.2 \mu \mathrm{m}$ \\
\hline Au Thickness & $300 \AA$ & $200 \AA$ & $300 \AA$ \\
\hline
\end{tabular}

III. X-RAY RESPONSE

The detectors were evaluated using an $55 \mathrm{Fe}$ source and tunable $x$-rays in the $1.5-2.5 \mathrm{keV}$ range from an $x$-ray tube equipped with a monochromator. Figure 2 shows ${ }^{55} \mathrm{Fe}$ spectra from detector \#4-3 at different operating voltages. Two distinct background features can be seen in these spectra: a higher level step-like background extending from the full energy peak to a lower energy threshold and a low level background which extends from this threshold to the lowest energy measurable 


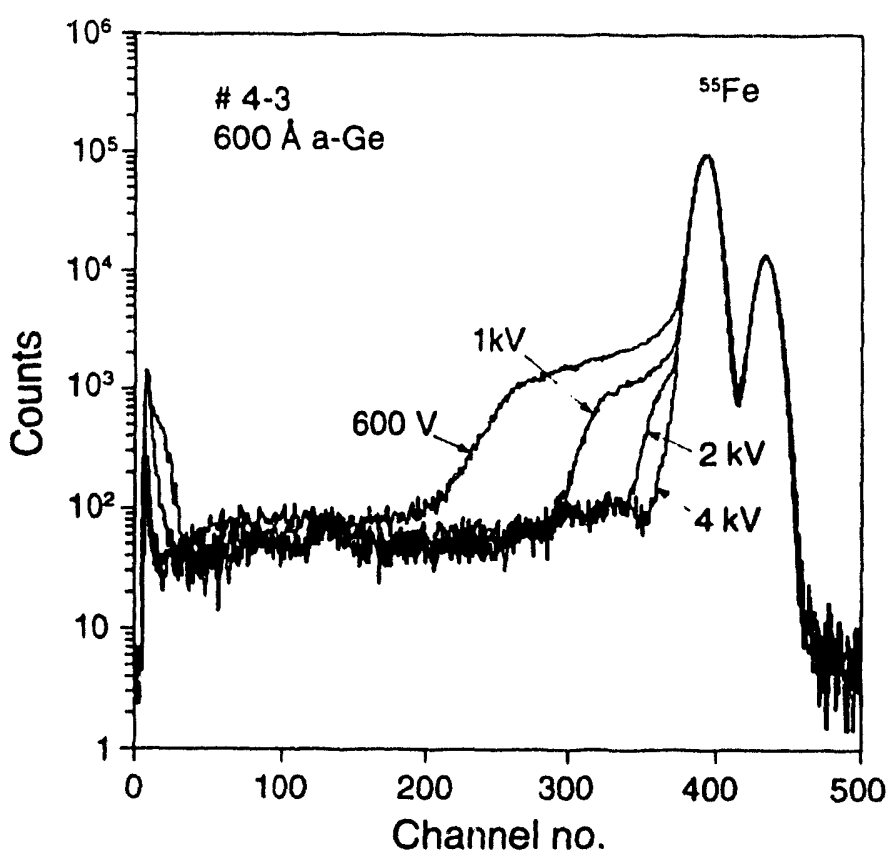

Fig. 2. ${ }^{55} \mathrm{Fe}$ spectra from a Ge detector with a-Ge contact under different applied voltages.

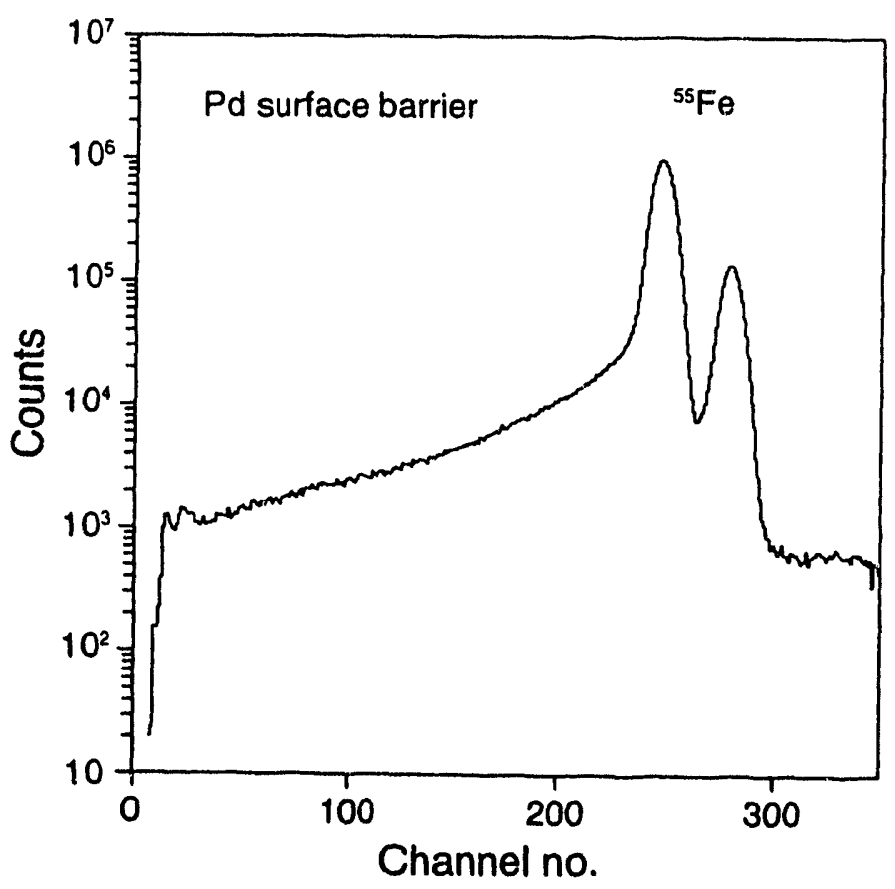

XBL $9310-4353$

Fig. 3. ${ }^{55} \mathrm{Fe}$ spectrum from a Pd surface barrier Ge detector.

above the electronic noise. The integrated counts in the low level background remains constant with change in bias voltage, while the threshold energy of the step background shifts towards the full energy peak with increasing applied voltage and is virtually eliminated at sufficiently high voltages. It is interesting to compare these results with those from detectors with different types of contacts. Figure 3 shows an ${ }^{55} \mathrm{Fe}$ spectrum obtained with a Pd surface barrier Ge detector, which exhibits a high level background extending to the noise level. Figure 4 shows spectra obtained from detectors with B implanted contacts made under different implant conditions [3]. (The $\mathrm{BF}_{2}$ and $\mathrm{BF}_{4}$ implantations at $25 \mathrm{keV}$ yield ranges in the sample equivalent to $B$ implantations at $5.6 \mathrm{keV}$ and $3.2 \mathrm{keV}$, respectively). These spectra exhibit step-like background features which vary with the implant conditions. In contrast to the a-Ge contact detector, the spectral background obtained with Pd surface barrier and B implanted contacts do not exhibit significant voltage dependence. For the a-Ge contact detector at $4000 \mathrm{~V}$ bias, the peak-to-background ratio (defined as the ratio of integrated counts in the full energy peak to those in the low level background) is 56, compared to 5.2 for the Pd surface barrier detector and 20 for the $25 \mathrm{keV} \mathrm{B}$ implant contact detector.

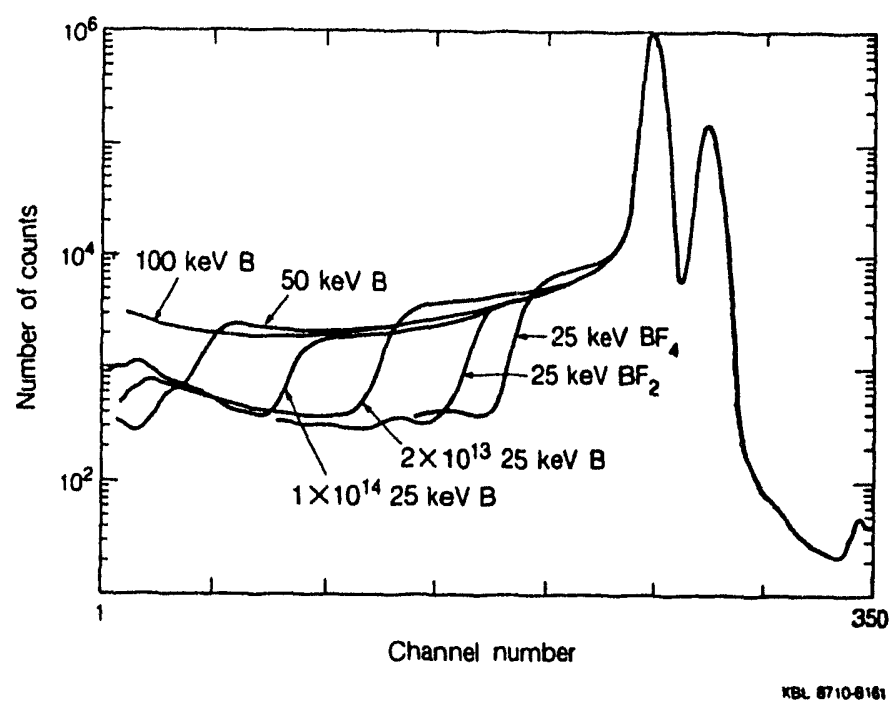

Fig. 4. ${ }^{55} \mathrm{Fe}$ spectra from Ge detectors with B ion implanted contacts produced under different implantation conditions.

Figure 5 compares the low energy $x$-ray performance of detectors \#8-2 and \#6-6, which have a-Ge contacts of different thicknesses. The $x$-rays were obtained from a monochromator tuned to the $1.77 \mathrm{keV} \mathrm{M} \mathrm{X}$-rays from tungsten plated on a copper anode $x$-ray tube. The applied voltages were chosen such that the electric field at the a-Ge contact for both detectors was approximately the same $(\sim 1400 \mathrm{~V} / \mathrm{cm})$. These spectra have been normalized with respect to the integrated counts in the photopeak of the third harmonic $(7.08 \mathrm{keV})$ output of the monochromator, such that the large difference in intensity at $1.77 \mathrm{keV}$ is due to the absorption of the low-energy $x$-rays in the a-Ge contacts. As can be seen from Fig. 5, the detector with the thicker a-Ge contact $(1.2 \mu \mathrm{m}$ compared with 0.12 $\mu \mathrm{m})$ is much less efficient at $1.77 \mathrm{keV}$ than the detector with the thin a-Ge contact. The relative transmission of the thick aGe compared with the thin a-Ge (including the metal layer) is $-12 \%$, in good agreement with the calculated value of $10 \%$. This indicates that the a-Ge layer behaves as a true dead layer from which no signal carriers are collected. The small peak at 
$1.2 \mathrm{keV}$ in the spectrum from the thick a-Ge contact detector is due to $\mathrm{Ge} \mathrm{L}$-rays fluoresced from the a-Ge layer and detected in the active volume of the detector. This is further evidence that the a-Ge layer acts as a passive layer. This same fluorescence peak from the thin a-Ge contact is too weak to be seen. The peak at approximately $0.6 \mathrm{keV}$ observed in both spectra is the Ge L escape peak of the $1.77 \mathrm{keV}$ line. The low L fluorescence yield $(<1 \%)$ accounts for the small intensities of these peaks. These spectra also display low level backgrounds extending from the photopeak to the electronic noise levels. There are, however, no clearly discemible step-like background features as seen in the ${ }^{55} \mathrm{Fe}$ spectra (Fig. 2) but these may have merged into the main peak because of the lower relative energy resolution. The asymmetry observed in the main peak in the thin a-Ge spectrum is likely a manifestation of this effect, while the thick a-Ge detector displays a symmetrical photopeak. The reason for this difference in symmetry is not clear. For the thick a-Ge contact detector, the photopeak resolution is $156 \mathrm{eV} \mathrm{FWHM}$ and the electronic noise is $142 \mathrm{eV}$ FWHM. This gives an intrinsic resolution of $65 \mathrm{eV}$ FWHM, equivalent to a Fano factor of 0.14 .

The improvement in low-energy $x$-ray response of both detectors of Fig. 5 is dramatic when compared with the result obtained with a Pd surface barrier Ge detector (Fig.6). The peak-to-background ratio is 29 for the a-Ge contact detectors while only 2.2 for the Pd surface barrier detector. The Ge detectors with a-Ge contacts also compare very favorably with $\mathrm{Si}(\mathrm{Li})$ detectors at these low energies, as can be seen by comparing Fig. 5 with Fig. 7, which shows a $2.0 \mathrm{keV}$ spectrum from a $\mathrm{Si}(\mathrm{Li})$ detector with a $\mathrm{Pd}$ surface barrier contact.

\section{DISCUSSION}

The spectral backgrounds observed in semiconductor detectors are due to incomplete charge collection from $x$-ray interactions that occur close to the entrance contacts of the detectors. Various mechanisms have been proposed to account for this effect, such as the escape of photoelectrons from the active volume of the detector [4], the loss of carriers at contacts as a result of charge expansion due to carrier diffusion [5] or warm-carrier escape [6], trapping at bulk defect sites [7], and combinations of these factors $[8,9]$. The two distinct background features observed for detectors with a-Ge contacts lead to the conclusion that there are two different mechanisms involved and it is natural to examine both photoelectron escape and charge expansion as causes of the background.

Figure 8 shows schematically what occurs when a low energy $x$-ray photon interacts in a semiconductor detector. Initially, a photoelectron is ejected from an atomic shell $(\mathrm{L}$ or $\mathrm{K})$ followed by de-excitation via the emission of either an Auger electron or a fluorescence photon. The fluorescence photon can be reabsorbed within the detector or it can escape which will contribute to the escape peak in the spectrum. The photoelectron and Auger electron will travel within the crystal lattice, creating a cascade of energetic carriers and subsequently a distribution of free electrons and holes. The creation of free carriers is confined, on average, to a spherical volume with a radius equal to the larger of the photoelectron's or Auger electron's projected range $\left(R_{p}\right)$. Immediately after their creation, the cloud of carriers begins to expand due to warm carrier effects and/or diffusion. Under an electric field, the cloud of electrons and holes will drift in opposite directions as they expand.

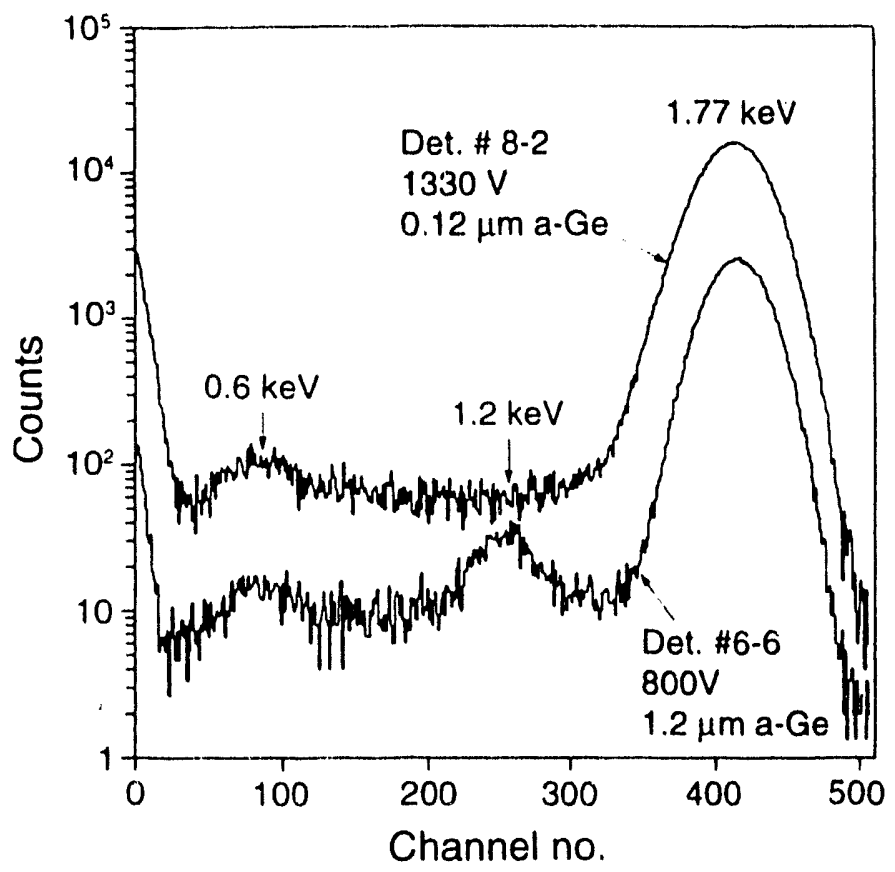

XBL 9310.4351

Fig. 5. $1.77 \mathrm{keV}$ spectra from Ge detectors with different a-Ge contact thicknesses.

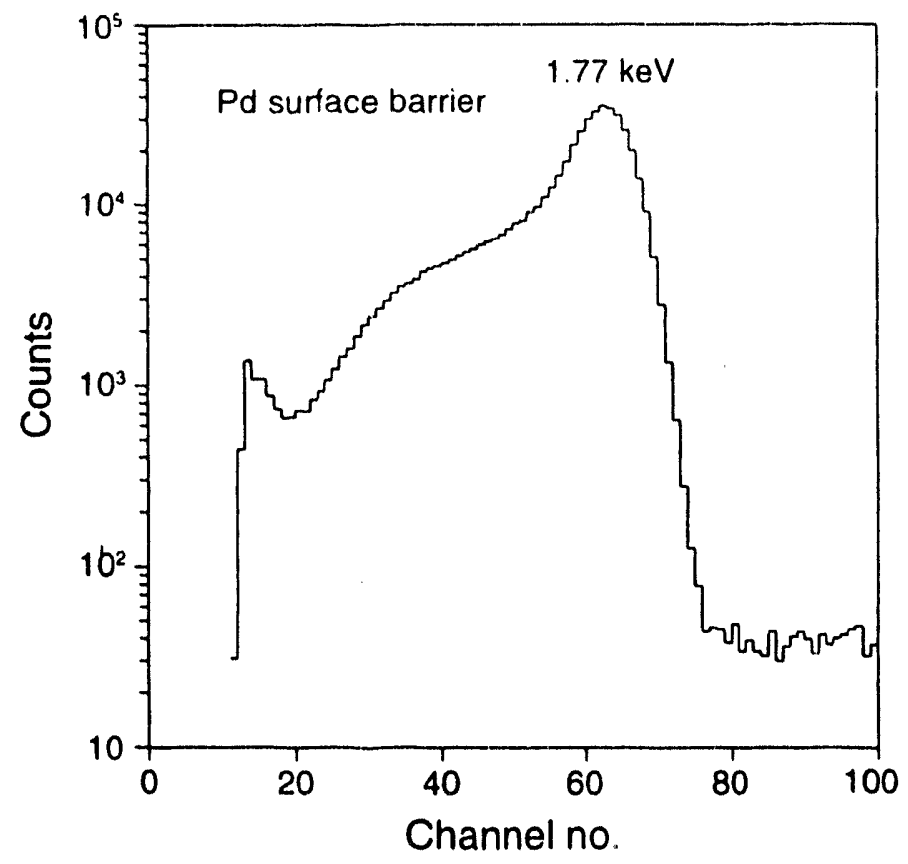

XBL 9310.435

Fig. 6. 1.77 keV spectrum from a Pd surface barrier Ge detector. 


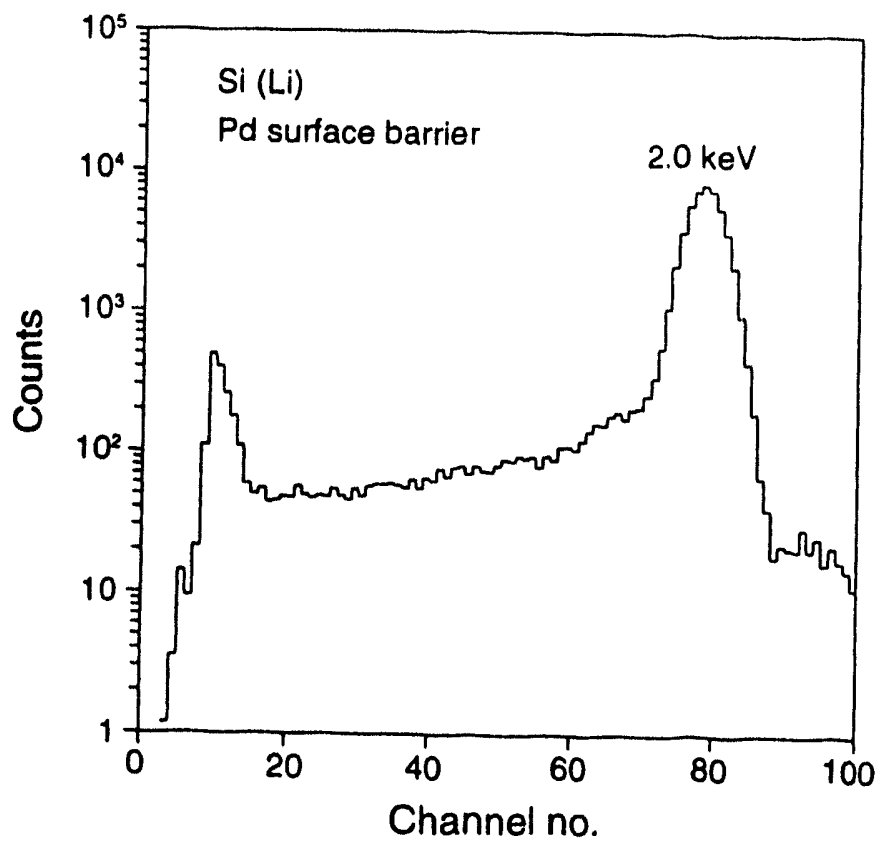

XBL $9310-4350$

Fig. 7. $2.0 \mathrm{keV}$ spectrum from a $\mathrm{Si}(\mathrm{Li})$ detector with a Pd surface barrier contact.

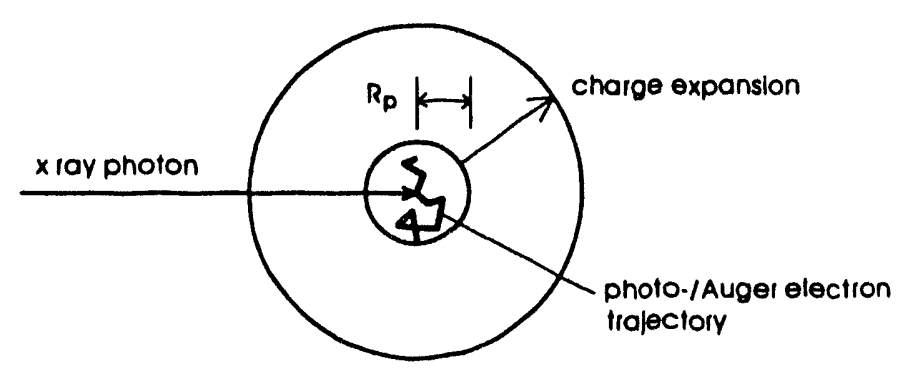

XBL 9310.144

Fig. 8. Events following the absorption of an $\mathrm{x}$-ray photon in a semiconductor detector.

By placing the $x$-ray interaction point at various depths in a detector and examining the consequences, it is possible, at least qualitatively, to determine the resulting spectral response. This is illustrated in Fig. 9 with the corresponding charge collection efficiency plotted as a function of depth. For the detectors under study, the $x$-rays are stopped within a very short distance from the entrance contact biased with a negative potential. Therefore, the detector signals are generated virtually entirely through the collection of electrons across the detector, and only the electron distributions are shown in Fig. 9. Loss of electrons from an absorption event will give rise to a reduced signal, contributing to the spectral background. The electric field resulting from the applied voltage is assumed to terminate abruptly at the a-Ge/crystalline $\mathrm{Ge}$ interface. When the $x$-ray interaction occurs in the a-Ge and the range of the photo- or Auger electron is confined in the a-Ge, the free carriers that are created will not spread significantly, since the carrier mobility and lifetime are very small in the highly disordered a-Ge [10]. With no electric field in the a-Ge layer, no carriers are collected and no signal is generated. If the interaction occurs within a distance $R_{p}$ from the $a-G e / c r y s t a l l i n e$ $\mathrm{Ge}$ interface, part of the carrier generation region extends into the crystalline side and the electron cloud generated there will expand due to the escape of warm electrons [6] or diffusion. The electrons that reach the a-Ge contact are assumed to be lost due to trapping and recombination. The rest of the electrons will be collected by the electric field giving rise to a reduced signal (i.e., a background count). The fraction of charge that is collected increases rapidly with increasing interaction depths. At depths where the charge generation region is fully contained in the crystalline side, charge loss occurs only as a result of charge expansion which causes some of the electrons to reach the a-Ge contact in opposition to their drift in the electric field. In this regime, the rate of increase of charge collection efficiency as a function of depth becomes slower. Full charge collection occurs at depths where the electron cloud is collected by the electric field before any of the electrons reach the a-Ge contact. With increasing electric field strength, the electron cloud, as it expands, is pulled more strongly away from the a-Ge contact, and the depth over which incomplete charge collection occurs becomes shallower. This effect is represented by the dashed lines of Fig. 9.
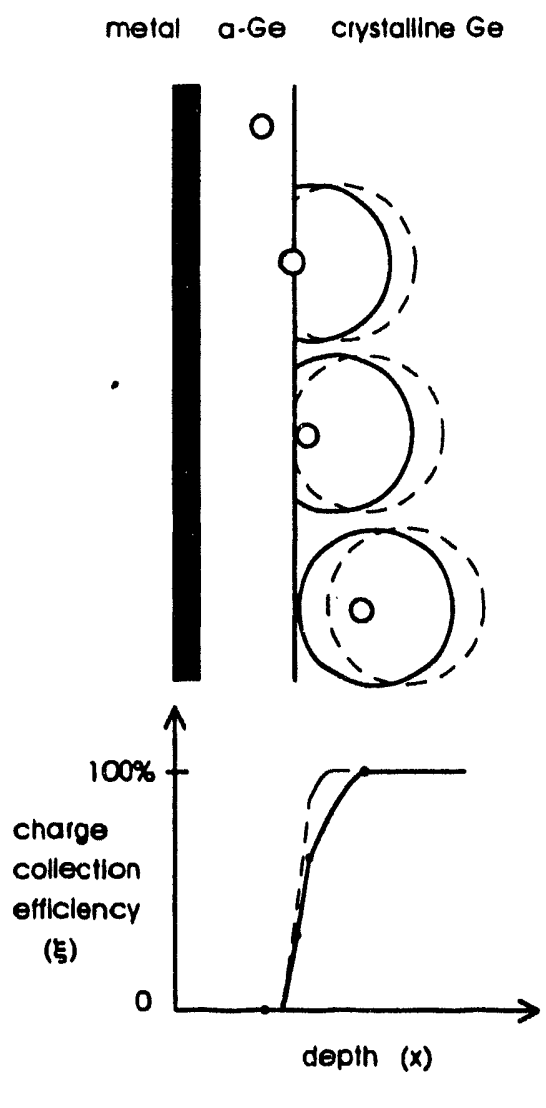

Fig. 9. Variation of charge collection efficiency with the depth of $x$-ray interaction in an a-Ge contact detector. 
The spectral response is related to the charge collection efficiency $(\xi)$ and the lincar absorption coefficient $(\mu)$ for the particular $x$-ray photon energy. The number of counts per unit energy can be writuen as:

$$
\frac{d N}{d E}=\frac{N_{0}}{E_{0}} \mu e^{-\mu x} / \frac{d \xi}{d x}
$$

where $N_{0}$ is the total number of $x$-ray photons absorbed in the detector, and $E_{0}$ is the energy of the $x$-ray photon. The actual spectral response is the convolution of this expression with the detector resolution which includes the contributions from charge statistics and electronic noise. The number of counts per unit energy is inversely proportional to the slope of the charge collection efficiency curve. An abrupt change in slope will result in a step-like background feature in the spectrum as observed in the ${ }^{55} \mathrm{Fe}$ spectra. The electron range effect gives rise to the steep part of the charge collection efficiency curve and is responsible for the observed low-level background. The charge expansion effect gives rise to the slower increase and is responsible for the higher level step background. The threshold energy of the step background is related to the charge collection efficiency at the depth where the charge generation region is just fully contained in the crystalline bulk. With increasing applied voltages, more electrons are collected at this depth and the threshold shifts to higher energy. Also, full charge collection takes place at a shallower interaction depth, resulting in a reduced number of integrated counts in the step background.

According to this model, the thickness of the region inside the detector which gives rise to the low-level background should be about $2 R_{p}$, if the a-Ge layer thickness is larger than $R_{p}$. If the a-Ge thickness is less than $R_{p}$, it should be $R_{p}$ plus the a-Ge layer thickness plus the Ge-equivalent contribution from the Au layer. The electron range can be calculated using an empirical formula which has been fitted to experimental data [11]. For $5.9 \mathrm{keV}$ photons, the resulting photoelectrons have an energy of $4.7 \mathrm{keV}$ and their projected range in $\mathrm{Ge}$ is $0.23 \mu \mathrm{m}$. Adding to this value the a-Ge thickness of $0.06 \mu \mathrm{m}$ and an estimated $0.15 \mu \mathrm{m}$ Ge-equivalent contribution from the $\mathrm{Au}$ layer yields a total of $0.44 \mu \mathrm{m}$. For $1.77 \mathrm{keV}$ photons, the projected range of the $1.2 \mathrm{keV}$ Auger electrons, which have a higher energy than the photoelectrons, is $0.012 \mu \mathrm{m}$. Since this is less than the thickness of the a-Ge layer, the incomplete charge collection layer thickness should be twice the projected range, i.e. $0.024 \mu \mathrm{m}$. The incomplete charge collection layer thicknesses, calculated using known $x$-ray absorption co fficients and the peak-to-background ratios obtained from the 5.9 and $1.77 \mathrm{keV}$ spectra, are $0.39 \mu \mathrm{m}$ and $0.03 \mu \mathrm{m}$, respectively. The agreement between the two sets of values for the incomplete charge collection layer thickness is excellent, considering the simple assumptions used. On the other hand, the high-level step background component is difficult to model quantitatively, as it requires a detailed knowledge of many factors which are not known or cannot be readily calculated, such as the energy distribution of the carriers after their creation, shielding of the electric field by the electron-hole plasma, and the recombination velocity at the a-Ge/crystalline
Ge imerface. Nevertheless, if the loss of carriers due to charge expansion is indeed the mechanism responsible for the step background observed in the a-Ge contact detectors, then this mechanism is strongly affected by the electric field. Therefore, other processes will need to be invoked to explain the origin of the spectral background observed in detectors with other types of contacts which do not exhibit a significant voltage dependence.

In the case of B implanted contacts, although the spectra look very similar to that of the a-Ge contact detector at low bias, there is no significant dependence on applied voltage but there is a strong dependence on implantation parameters. In this case, the contact itself constitutes an incomplete charge collection layer. Although the contact layer is heavily doped and radiation damaged by the implantation process, the carrier lifetime and mobility are apparently sufficiently high that electrons generated within this layer have a finite probability of diffusing out of the contact and being collected. Since there is no electric field present inside the contact, the diffusion process and thus the resulting spectral backgrounds do not exhibit a significant voltage dependence. On the other hand, higher implant energies result in thicker contacts and thus thicker incomplete charge collection layers (i.e., a lower peakto-background ratio). Also, as the contact thickness increases, the collection efficiency for carriers created near the surface decreases, shifting the threshold energy of the step background to lower energies. The two spectra corresponding to contacts formed with the same implant energy but different dosage illustrate that the lower dosage implant results in a higher collection efficiency and thus a higher threshold energy because of the reduced doping and damage. However, the integrated counts in the step background are roughly equal since the thicknesses of the two contacts are the same. The low-level backgrounds are again attributed to the electron range effect which takes place within a distance $R_{p}$ from the crystal surface.

Based on the contact structure, the spectral response of the Pd surface barrier detector is expected to be similar to the a-Ge contact detectors, with $\mathrm{Pd}$ in the role of the a-Ge. Instead, the resulting spectrum exhihits a high-level continuum background with no significant dependence on applied voltage. One possible explanation is that a high concentration of p-type defects is formed in the Ge next to the Pd contact, creating a region free of electric field. As in the case of the B implant contacts, loss of signal carriers takes place in this region as the electrons diffuse out to the defect-free region where they can be collected by the electric field. Further investigations are needed to verify this hypothesis.

\section{NOISE CONSIDERATIONS}

Electrical conduction in a-Ge is characterized as hopping conduction with a temperature dependence of $\exp \left(\mathrm{T}^{-1 / 4}\right)$. At liquid nitrogen temperatures, the resistivity of the a-Ge film used in this investigation was measured to be $\sim 10^{8} \Omega-\mathrm{cm}$. Depending on the geometry of the a-Ge contact and the particular application, the resulting resistance of the a-Ge film may contribute significant noise to the electronic system. For the 
detectors studied here, which have an active area of $0.2 \mathrm{~cm}^{2}$, the resistance of the a-Ge film ranged from $3 \mathrm{k} \Omega$ for the 600 $A$ thick film to $60 \mathrm{k} \Omega$ for the $1.2 \mu \mathrm{m}$ film. Assuming only Johnson noise contributions, they would generate voltage noise densities of $6 \mathrm{nV} / \mathrm{Hz}^{1 / 2}$ to $27 \mathrm{nV} / \mathrm{Hz}^{1 / 2}$. This noise source is, however, shunted by the capacitance of the thin a-Ge layer. The resulting RC time constant, which depends only on the resistivity of the material, is $140 \mu \mathrm{sec}$. Therefore, for typical amplifier peaking times of 1 to $10 \mu \mathrm{sec}$, the noise is strongly attenuated and becomes negligible for the thin layers. Noise from thick a-Ge layers may become dominant, especially if the detector system is operated at long peaking times. This would typically not be an important consideration since thin a-Ge layers are desirable to minimize photon absorption at the contact.

\section{CONCLUSION}

Ge detectors with a-Ge contacts have been evaluated using low energy $x$-rays. Their spectral response exhibits a step-like background feature which shows a strong dependence on applied voltage. This background feature can be completely suppressed with high operating voltages, leaving only a lowlevel continuum background. The spectral response at high voltages is superior to those obtained using detectors with $\mathrm{Pd}$ surface barrier or B implanted contacts, and also compares very favorably with $\mathrm{Si}(\mathrm{Li})$ detectors. In the a-Ge contact detectors, the a-Ge layer acts as a true dead layer, in which no charge collection takes place. The apparent incomplete charge collection layer observed is not due to any physically distinguishable region in the detector, but is attributed to the effects of electron range and charge expansion which allow signal carriers generated within this apparent layer to be lost at the a-Ge contact. The thickness of the incomplete charge collection layer varies, depending on the range of the photoelectron or Auger electron generated by the absorbed photon, and on the electric field near the contact. In contrast, the backgrounds observed in detectors with B implant contacts and Pd surface barrier contacts can be explained by the existence of a physical layer in the Ge crystal which is free of electric field due to the presence of a high concentration of dopant or defects, and in which charge loss can take place.

\section{REFERENCES}

[1] P.N. Luke, C.P. Cork, N.W. Madden, C.S. Rossington and M.F. Wesela, "Amorphous Ge Bipolar Blocking Contacts on Ge Detectors," IEEE Trans. Nucl. Sci., vol. 39, no. 4, pp. 590-594, Aug. 1992.

[2] C.S. Rossington, R.D. Giauque and J. M. Jaklevic, "A Direct Comparison of $\mathrm{Ge}$ and $\mathrm{Si}(\mathrm{Li})$ Detectors in the 2-20 keV Range," IEEE Trans. Nucl. Sci., vol. 39, no. 4, pp. 570 576. Aug. 1992.

[3] R.E. McMurray, Jr., P.N. Luke, J.M. Jaklevic and J.T. Walton, "Effects of Germanium Detector Contacts on Dead Layer Performance for Soft X-rays," presented at the Stanford Synchrotron Radiation Workshop, Oct. 1987, unpublished.

[4] Y. Inagaki, K. Shima and H. Maezawa, "Response Functions of a $\mathrm{Si}(\mathrm{Li})$ Detector for 1.3.4.0 keV Monochromatic
Photons," Nucl. Instr. Meth., vol. B27, pp. 353-359, July 1987

[5] F.S. Goulding, "Some Aspects of Detectors and Electronics for X-ray Fluorescence Analysis," Nucl. Instr. Meth., vol. 142. pp. 213-223, April 1977.

[6] J. Llacer, E.E. Haller and R.C. Cordi, "Entrance Windows in Germanium Low-energy X-ray Delectors," IEEE Trans. NuCl. Sci., vol. NS-27, pp. 247-251, Feb. 1980.

[7] M. Geretschlager, "Monte Carlo Simulation of the Response of $\mathrm{Si}(\mathrm{Li}) \mathrm{X}$-ray Detectors to Proton Induced $\mathrm{K} \mathrm{X}$-rays of Light Elements Applied to Efficiency Determination," Nucl. Instr. Meth., vol. B28, pp. 289-298, Sept. 1987.

[8] S. Goto, "Response Functions of a $\mathrm{Si}(\mathrm{Li})$ Detector for Photon Energies from 1 to $10 \mathrm{keV}, "$ Nucl. Instr. Meth., vol. A333, pp. 452-457. Sept. 1993.

[9] J. X. Wang and J. L. Campbell, "Monte Carlo Simulation of the Response of $\mathrm{Si}(\mathrm{Li})$ Detectors to Monoenergetic X-rays," Nucl. Instr. Meth., vol. B54, pp. 499-506, April 1991.

[10] T.D. Moustakas and W. Paul, "Transport and Recombination in Sputtered Hydrogenated Amorphous Germanium," Phys. Rev., vol. 16, no. 4, pp. 1564-1576, Aug. 1977.

[11] C. Feldman, "Range of 1-10 keV Electrons in Solids," Phys. Rev., vol. 117, no. 2, pp. 455-459, Jan. 1960. 

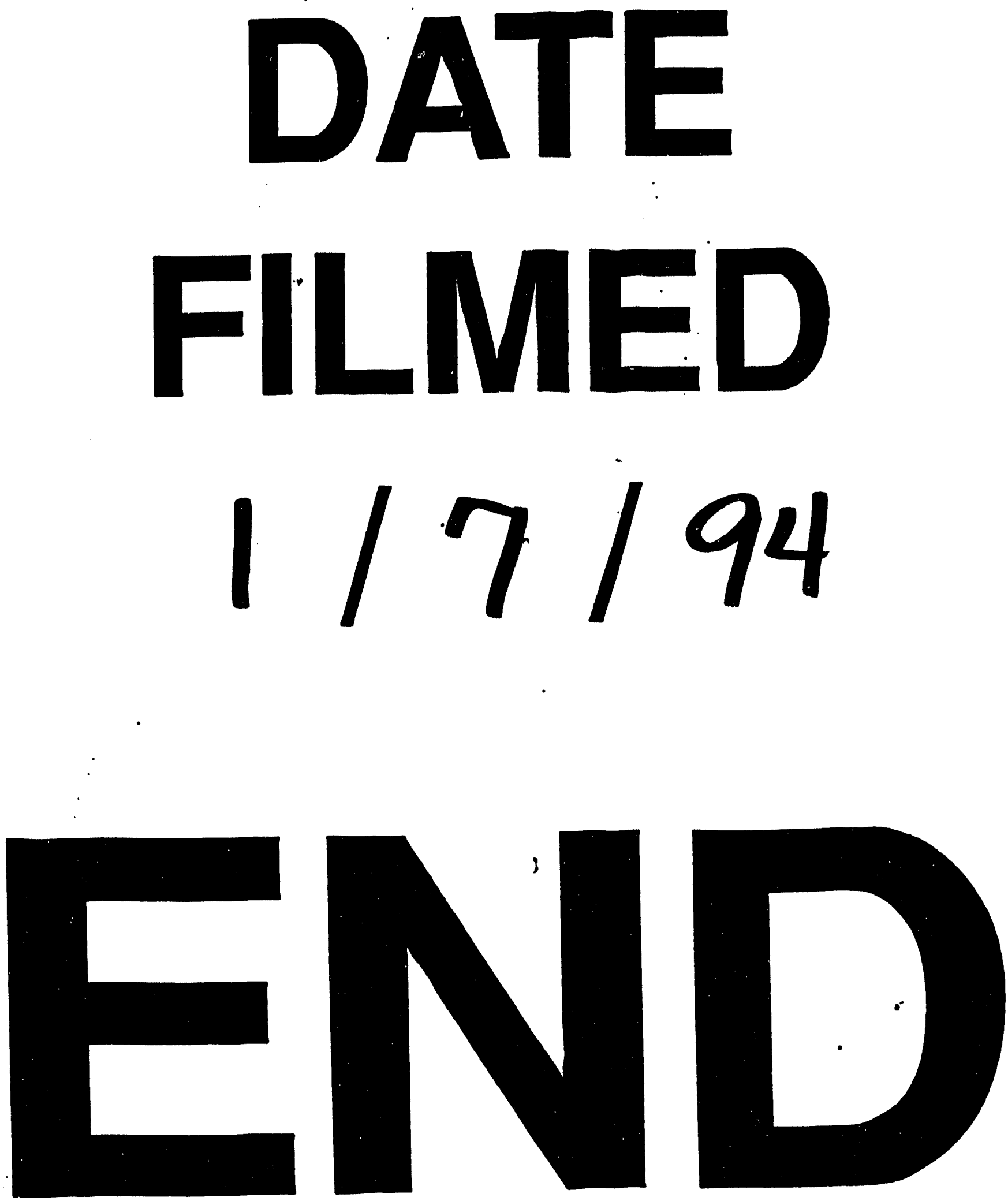

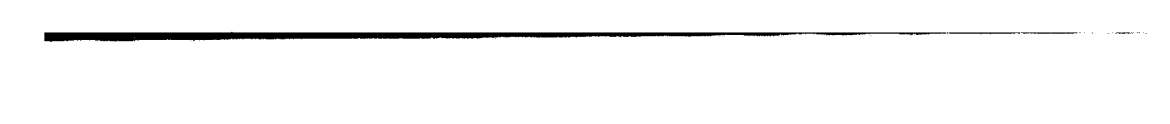